\title{
Paraguay: Crecimiento económico, conflicto social E INCERTIDUMBRE POLÍTICA
}

\author{
Paraguay: Economic growth, social conflict and political uncertainty
}

\section{DIEGO ABENTE-BRUN}

\begin{abstract}
RESUMEN
El presente trabajo analiza la coyuntura paraguaya centrándose en tres dimensiones principales. La primera es la económica. Se resalta la altísima tasa de crecimiento alcanzada después de la crisis de 2008, la estabilidad de precios, el buen manejo fiscal, el alto nivel de reservas, el bajo grado de endeudamiento $\mathrm{y}$, en general, las buenas perspectivas económicas.

La segunda dimensión es la social. En este plano se examinan tres conjuntos de problemas. En primer lugar se enfatiza la escasa capacidad de generación de empleos de una economía excesivamente dependiente de los agronegocios, especialmente la soja y la carne. En segundo lugar se constata que a pesar de haberse realizado ingentes inversiones en programas sociales los niveles de pobreza no han disminuido y la distribución de los ingresos ha empeorado. Finalmente, se discute la problemática del acceso a la tierra y se concluye que, lamentablemente, a pesar de haber sido esta la bandera insignia de la campaña de Lugo, casi cuatro años después de su asunción hay muy poco en su haber. La tercera dimensión, la política, analiza la lógica del gobierno y la oposición a medida que ambas se preparan para la contienda presidencial del 21 de abril de 2013 . Se observa que el proceso que comenzó en el gobierno con inmensas dificultades y graves errores, se encuentra sin embargo encaminándose mejor. En contraste, el panorama en el Partido Colorado, que parecía claro un año atrás, se ve cada vez más incierto.
\end{abstract}

Palabras clave: Economía, empleos, pobreza, gobierno, oposición.

\begin{abstract}
This article analyzes the Paraguayan situation focusing on three key dimensions. The first is the economic one. The article underlines the very high rates of growth achieved after the 2008 crisis, the low level of inflation, the solid management of the fiscal variables, the high level of reserves, the low debt ratio, and, in general, the very good economic prospects.

The second dimension is the social one. Three sets of problems are examined. First, the article notes the very weak job creation capability of an economy excessively dependent on agribusiness, especially soybeans and meat. Second, it stresses the fact that in spite of largescale investment in social policies poverty levels remain high and income distribution has worsened. Finally it discusses the problem of access to arable land for peasants and it notes that, unfortunately, in spite of having been the most emblematic point of its platform, after almost four years the government has little progress to show.

The third dimension, the political one, analyzes the government and the opposition as they prepared for the upcoming general elections scheduled for April 21, 2013 It notes that while the Government began in apparent disarray and making serious mistakes, things appear to be taken a better turn. Conversely, the panorama in the Colorado party that began as all clear, said, and done, has begun to unravel.
\end{abstract}

Key words: Economy, jobs, poverty, government, opposition. 
El año 2011 fue caracterizado a la vez por una tasa de crecimiento histórico, un incremento de la conflictividad social, e incertidumbre política. Luego de la crisis de 2008, que llevó a una caída del PIB del orden del 3,8 por ciento en 2009, las efectivas políticas anticíclicas produjeron una recuperación económica en 2010 cuando Paraguay alcanzó la tasa de crecimiento más alta de su historia, 15 por ciento, guarismo superado sólo por Qatar. En 2011, el crecimiento fue más moderado y la tasa fue del 4 por ciento.

A pesar del alto crecimiento económico, en el plano social los conflictos se incrementaron, especial pero no únicamente en torno a la problemática del acceso a la tierra y la polémica en torno a la "sojización" de la agricultura paraguaya y el rol de los llamados "brasiguayos" en la misma.

En el plano político, el gobierno logró despejar el constante acoso opositor que amenazaba con un juicio político. Pero si el panorama se aclaró en dicho sentido, una nueva lógica política empezó a desarrollarse en torno a las elecciones generales previstas para el 21 de abril de 2013. Gobierno y oposición entraron en un proceso temprano y traumático en búsqueda de una fórmula de victoria para las elecciones de 2013.

Hacia finales del año la confusión iba in crescendo. La predicción de los observadores es que el 2012 será un año complicado y problemático.

\section{EL CRECIMIENTO ECONÓMICO}

Luego de sufrir las consecuencias de la crisis internacional, la economía paraguaya se recuperó con fuerza inusitada en 2010 y mantuvo el ritmo hasta mediados de 2011. En el segundo semestre del año la aparición de un brote de fiebre aftosa en el Departamento de San Pedro afectó adversamente las exportaciones cárnicas y el sector de la industria frigorífica. Con posterioridad, una sequía prolongada castigó la producción agrícola y augura un año 2012 de menor crecimiento aunque las previsiones son aún muy preliminares.

La inflación se mantuvo bajo control disminuyendo del 7,2 por ciento interanual a diciembre de 2010 a 4,9 por ciento interanual a diciembre $2011 .{ }^{1}$ Las cuentas externas mostraron también un saldo favorable pese al déficit de la balanza comercial gracias a los ingresos de las binacionales Itaipú y Yacyretá, las remesas de los paraguayos en el exterior, y la afluencia de capitales externos.

El comportamiento de la política fiscal ha sido sólido. En 2011 Paraguay cerró el octavo año consecutivo de superávit fiscal primario, 1,4 por ciento del PIB. La deuda externa disminuyó del 18 por ciento del PIB en 2007 a 9,8 por ciento en 2011. La formalización de la actividad económica siguió avanzando a pasos firmes, lo que se tradujo en el aumento del número de contribuyentes registrados en la Administración Tributaria, de 429.881 
Cuadro 1: Crecimiento del PIB por sectores

\begin{tabular}{lcrrr}
\hline Sectores & 2008 & 2009 & 2010 & 2011 \\
\hline Primario & 9,2 & $-17,2$ & 34,1 & 4,0 \\
Secundario & 2,0 & 0,8 & 6,3 & $-0,8$ \\
Terciario & 4,8 & 2,2 & 9,0 & 5,7 \\
Total & 5,8 & $-3,8$ & 15,0 & 4,0 \\
\hline
\end{tabular}

Fuente: Banco Central del Paraguay, Informe Económico Preliminar 2011, p. 54.

en 2007 a 586.633 en 2011, con incrementos del 9, 8,5 y 5,1 por ciento respectivamente en los tres últimos años. ${ }^{2}$

Estos resultados económicos extraordinariamente positivos crearon un ambiente de optimismo en el gobierno y el sector empresarial que lamentablemente no pudieron ser capitalizados en todo su potencial debido a conflictos sociales latentes y una coyuntura política enredada. A esto se agrega que se pronostica que el año 2012, último de este gobierno, el país podría de nuevo caer en recesión debido a la fuerte sequía del último semestre, el rebrote de la fiebre aftosa a fines de 2011, y las trabas comerciales impuestas por Argentina a las exportaciones paraguayas.

\section{CONFLICTOS EN EL FRENTE SOCIAL}

Como el motor principal del crecimiento ha sido nuevamente el sector capital-intensivo de la soja y la carne, las altas tasas de crecimiento se reflejaron sólo parcialmente en el empleo. Así la tasa de desempleo de 2011 para Asunción y la región central que concentra más del 60 por ciento de la población económicamente activa urbana se situó en torno al 7 por ciento, similar a la del 2010, y la del subempleo aumentó ligeramente de 23,9 por ciento a 24,5 por ciento. ${ }^{3}$

Similar en líneas generales fue el comportamiento de los porcentajes de pobreza. En 2010 el 34,7 por ciento de la población se encontraba por debajo de la línea de la pobreza y 19,4 por ciento vivía en la indigencia. La pobreza, como es de esperar, afecta con mayor fuerza a la población rural.

La evolución de la pobreza en los últimos tres años ha sido contradictoria. El total de la población bajo la línea de pobreza disminuyó de 37,9 por ciento en 2008 a 34,7 por ciento en 2010. Esta reducción obedece a la disminución drástica de la pobreza urbana entre 2008 y 2009, pues entre 2009 y 2010 los guarismos cambiaron poco. Además, la 
Cuadro 2: Población bajo la línea de pobreza e indigencia 2010

\begin{tabular}{lcc}
\hline & Porcentaje de pobres & Porcentaje de indigentes \\
\hline Total país & 34,7 & 19,4 \\
Total urbano & 24,7 & 10,3 \\
Total rural & 48,9 & 32,4 \\
\hline
\end{tabular}

Fuente: DGEEC, Resultados Principales de Pobreza y Distribución del Ingreso, 2010, Asunción, Paraguay, p. 6.

Cuadro 3: Evolución de la pobreza y de la indigencia 2008-2010

\begin{tabular}{lccc}
\hline & $\begin{array}{c}2008 \\
\text { Pobreza (indigencia) } \\
\text { Porcentaje }\end{array}$ & $\begin{array}{c}2009 \\
\text { Pobreza (indigencia) } \\
\text { Porcentaje }\end{array}$ & $\begin{array}{c}2010 \\
\text { Pobreza (indigencia) } \\
\text { Porcentaje }\end{array}$ \\
\hline Total país & $37,9(19,0)$ & $35,1(18,8)$ & $34,7(19,4)$ \\
Total urbano & $30,2(10,6)$ & $24,7(9,3)$ & $24,7(10,3)$ \\
Total rural & $48,8(30,9)$ & $49,8(32,4)$ & $48,9(32,4)$ \\
\hline
\end{tabular}

Fuente: DGEEC, Resultados Principales de Pobreza y Distribución del Ingreso, 2010, Asunción, Paraguay, p. 7.

pobreza rural y la indigencia, rural y urbana, aumentaron ligeramente. El Cuadro 3 ilustra esta situación.

Aunque el periodo bajo consideración es corto, estos resultados permiten hacer dos reflexiones. En primer lugar la disminución de la pobreza urbana en más de cinco puntos porcentuales entre 2008 y 2009 resulta curiosa, puesto que en 2009 la economía se contrajo en 3,8 por ciento y los programas sociales recién estaban empezando y no estuvieron direccionados a las áreas urbanas.

En segundo lugar, los datos sugieren que algunos de los programas emblemáticos del gobierno han tropezado con problemas. Por ejemplo, el número de beneficiarios de transferencias directas con corresponsabilidades aumentó de cerca de 10.000 a aproximadamente 100.000 familias en extrema pobreza entre fines de 2008 y 2010. Un aumento tan drástico en tan corto tiempo debería poder observarse en las estadísticas de indigentes. Sin embargo, el porcentaje de la población rural en extrema pobreza, target privilegiado de esta política, se mantuvo constante entre 2009 y 2010 y aumentó en el área urbana.

Finalmente, tampoco se logró progresar significativamente en la reducción de la desigualdad aunque este indicador se manifiesta con considerable rezago. El coeficiente de Gini aumentó de 0,506 a 0,512 como muestra el Cuadro 4 a continuación:

Pero más allá del problema del empleo y la pobreza, la tensión social se centró como desde hace tiempo en el problema del acceso a la tierra. La campaña de Lugo había hecho de la reforma agraria su bandera principal en el campo de la política domestica, pero los 
Cuadro 4: Coeficiente de Gini 2008-2010

\begin{tabular}{llll}
\hline Coeficiente de Gini & 2008 & 2009 & 2010 \\
\hline Total país & 0,506 & 0,487 & 0,512 \\
Total urbano & 0,453 & 0,423 & 0,459 \\
Total rural & 0,557 & 0,554 & 0,558 \\
\hline
\end{tabular}

Fuente: DGEEC, Resultados Principales de Pobreza y Distribución del Ingreso, 2010, Asunción, Paraguay, p. 16.

años fueron pasando sin progresos significativos. El Indert, el instituto encargado de la reforma agraria, es lo más parecido que existe a un barril sin fondo de corrupción y el gobierno, con excepción de la breve gestión del ingeniero Eugenio Alonso, poco o nada hizo por sanearlo. Al contrario, las viejas prácticas continuaron, y nuevos privilegiados se aprovecharon de la estructura ya montada para continuar con el mismo juego.

La corrupción en el Indert es polifacética e incluye entre otros mecanismos el pago sobrevalorado de "expropiaciones" destinadas a colonias agrícolas, la re-venta por parte de supuestos beneficiarios de los lotes agrícolas que luego vuelven a reclamar lotes, la adjudicación de lotes a personas que no son sujetos de la reforma agraria, la percepción de ingresos inferiores a los pactados por las ventas de lotes, y la doble y triple titulación de las tierras. Todos estos elementos en distinta combinación configuran el llamado problema de las "tierras mal habidas" que si bien se inició durante la dictadura de Stroessner que adjudicaba propiedades a sus generales y allegados ha continuado campante y sonante hasta la fecha. Según cálculos del Servicio Nacional de Catastro Paraguay posee fincas cuya superficie, sumada, excede en $150.000 \mathrm{~km}^{2}$ el territorio nacional. ${ }^{4}$

En el 2010 y a finales de 2011 dos casos emblemáticos simbolizaron esta crisis: el caso Teixeira y el caso Favero. El primero consistió en el intento de expropiación de entre 10 y 20 mil hectáreas de propiedad de un terrateniente brasiguayo, Ulisses Teixeira, en la zona central del país por parte de líderes campesinos afines al gobierno que deseaban ésa, y solo esa propiedad. La propiedad se encontraba, aparentemente, sobrevalorada y el señor Teixeira demostraba un inusitado interés en ser expropiado. Tras un escándalo

4 La cobertura sobre la corrupción en el Indert ha sido extensa y la institución no emitió un solo desmentido o aclaración. Como ejemplo véase, en ABC, las siguientes notas:

"No podrán recuperar muchas tierras públicas", 26-03-2012, "Denuncian compra irregular de 37.500 ha en el Chaco" 22-03-2012, "Pese a intervención, la mafia del Indert continúa operando", 22-03-2012, "La excusa de los inútiles" 1903-2012, “Conflicto será entre propietarios y no entre sintierras, dijo Camacho" 13-03-2012, Un empresario es apoderado de 15 "beneficiarios" de tierras del Indert" 12-03-2012.

Véase también en Última Hora las siguientes notas: "Firma de hija de Favero compró derecheras que suman 37 mil ha", 23-03-2012, "Indert paga USD 2,6 millones por una finca que costó USD 20.000" 21-03-2012, “El Indert adjudicó tierras fiscales a clubes sociales" 21-03-2012, "En el Indert se siguió robando durante el gobierno de Lugo," 15-03-2012, "Intervención: Desde Indert se crearon colonias fantasmas y se lotearon tierras del Estado," 15-03-2012, "Nadie sabe qué pasó con dinero de la venta de tierras públicas," 04-03-2012, "El Gobierno corrupto de las décadas del 70 y 80 rifó tierras en Ñacunday", 27-02-2012, 25 mil ha de tierras mal habidas en el Alto Paraná, sin recuperar", 26-02-2012, "El Gobierno debe investigar a fondo corrupción en el Indert", 24-02-2012, "La crisis de Nacunday desnuda enorme negociado en el Indert," 24-02-2012. 
que duró muchos meses el conflicto terminó, como tantos otros, en agua de borrajas. La propiedad sigue en manos de Teixeira. ${ }^{5}$

El segundo escándalo se produjo en la zona sur del país. El caso es sumamente curioso. Se trata de propiedades de entre 150.000 y 250.000 hectáreas en la zona más rica del país que fueron recobradas por el Estado por vía judicial en 1961, luego de un juicio de más de 40 años a los herederos de unas tierras compradas pero no abonadas y que el Estado recuperó en la década de 1920. Por razones inexplicables las tierras no fueron registradas a favor del Estado y hoy día se encuentran ocupadas en su mayoría por brasiguayos que la adquirieron del mayor terrateniente del país, el brasiguayo Tranquilo Favero, que a su vez alega haberlas comprado de su anterior propietario. Debido al caos de los registros de propiedad y la corrupción del Indert hoy en día no se sabe con certeza quién es dueño de qué, ni dónde están las tierras del Estado, ni si fueron vendidas, cuándo y a quién. ${ }^{6}$

En medio de esta confusión un grupo de campesinos liderados por Victoriano López se instaló en una propiedad supuestamente de Favero reclamando las tierras del Estado. El gobierno se encontró entre la espada y la pared. Por una parte, los campesinos, autodenominados "carperos", constituyen parte de su base de apoyo y contaban con la simpatía y respaldo de algunos altos personeros del gobierno. Por la otra, no tenía forma de demostrar de manera fehaciente la propiedad de las tierras en cuestión, ni si las mismas eran o no las famosas tierras no registradas de 1961, ni si habían sido vendidas o arrendadas. Para peor, varios líderes de la protesta adoptaron un tono xenófobo y los "brasiguayos" recurrieron al gobierno brasileño generándose así una tensión diplomática, aunque de baja intensidad.

A la fecha la solución está lejos de ser encontrada. Los "carperos" fueron reubicados en tierras supuestamente fiscales y la solución del problema no parece factible al menos en un tiempo razonable. El gobierno dispuso la "intervención" por cuarenta días del Indert, figura jurídica ambigua más mediática que efectiva, y designó al doctor Emilio Camacho, Asesor Jurídico de la Presidencia en tal carácter. Independientemente de la voluntad del Interventor es difícil que en tan corto tiempo el mismo dé solución a un problema que el gobierno en sus primeros tres años y medio de mandato no intentó solucionar.

En síntesis, lo que estos dos casos demuestran es una deficiencia de gestión, particularmente lamentable por tratarse de la demanda que la campaña de Lugo había convertido en emblemática. Es poco probable que en lo que le queda de tiempo al gobierno se puedan realizar avances significativos en este frente.

5 La cobertura del caso fue extensa e intensa y la oposición amenazó incluso con un juicio político al Presidente de procederse a la compra de las mismas. Como corolario véase Última Hora, 1 septiembre 2010, “Lugo anuncia hoy postura sobre el caso Teixeira".

6 El mejor tratamiento de este tema lo han hecho los periodistas Luis Bareiro y Patricia Vargas en una serie de artículos de investigación. Para un panorama general del caso véase, Última Hora, "El Estado solo vendió 12 por ciento de tierras recuperadas de Barthe", 1 octubre 2011. 


\section{INCERTIDUMBRE POLÍTICA}

\section{El efecto del estilo de liderazgo de Lugo}

La incertidumbre política respecto al 2013 transciende todas las fronteras partidarias. La alianza que llevó a Lugo al poder fue de carácter esencialmente electoral y no logró consolidarse como alianza política con proyección al futuro, como la Concertación en Chile. ${ }^{7}$ Eso hizo que los primeros tres años de gobierno hayan sido caracterizados por una lucha sin cuartel entre los diferentes componentes de la alianza en aras a posicionarse con vistas al 2013. El presidente Lugo demostró que tiene y sabe usar el poder pero su estilo de liderazgo no parece ser el apropiado para la construcción de un frente político amplio que permita consolidar los avances de su gobierno.

Lugo transmite la imagen de un Presidente que manda pero gobierna sólo por default. En primer lugar, al actuar como líder de las fuerzas de izquierda aglutinadas en el Frente Guasu dejó el liderazgo del PLRA al arbitrio de sus facciones internas. En segundo lugar, tampoco en el Frente Guasu ha ejercido un liderazgo claro, dejando que sus distintas facciones compitan entre sí con inusitada ferocidad por espacios de poder y por la cercanía a Lugo. El PLRA y el Frente Guasu saben que al final es el Presidente el que decide los cargos. Pero al reducir su rol a solo decidir los nombramientos ha dejado de ejercer liderazgo sobre lo más importante: la dirección del proceso y las prioridades de las políticas públicas. En este campo cada ministro o secretario imprime el rumbo que le parece mejor: los buenos ministros y secretarios hacen una buena gestión y los que no lo son pues no lo hacen. En tercer lugar, a fin de contrapesar al PLRA se ha rodeado de un grupo de colorados disidentes cuyo real poder electoral debe ser mínimo pues no cuentan con un solo cargo electivo. Este grupo, variopinto, ocupa importantísimas posiciones de poder y ha tomado medidas que en la práctica significan reivindicar al más represivo ex ministro del Interior de la dictadura stroenista y al autor intelectual de las violentas campañas de la misma contra opositores, todos los cuales sin distinción eran calificados de "comunistas apátridas".

De esta manera el liderazgo presidencial se ha desplazado del campo del proceso al campo de los cargos, especialmente aquellos con mayor poder de patronazgo o de mayor volumen de contratos públicos, pero en particular tres joyas de la corona, la petrolera estatal por la magnitud de compras y fletes que adjudica, la Dirección de Aduanas, y las binacionales Itaipú y Yacyretá por la gran discrecionalidad en el uso de sus recursos y su potencial de negociaciones financieras internacionales en temas de precios, deudas, contratos, etc.

7 Estrictamente hablando, la Alianza Patriótica por el Cambio dejó de existir como tal cuando el PLRA se retiró de la misma y la mayoría de los demás miembros decidieron conformar el Frente Guasu. Pero a los efectos de simplificar la discusión en este artículo seguiremos refiriéndonos a la Alianza como la suma del PLRA más el Frente Guasu, y el PDP, partido del ex ministro Rafael Filizzola. 


\section{El impacto de las elecciones municipales de noviembre de 2010}

La lectura de los resultados electorales de las elecciones municipales que sigue es dicotómica. Esta lectura dicotómica de los resultados electorales se justifica en Paraguay porque el sistema de partidos opera en clave bipartidaria; por una parte el Partido Colorado y por la otra los partidos no-Colorados. Por una buena parte de la historia desde el siglo XIX hasta mediados de la década de 1940 la clave fue bipartidaria: Partido Colorado versus Partido Liberal. Pero la larga hegemonía del Partido Colorado desde 1947 hasta 1989 y el control absoluto del Estado durante ese periodo le otorgó a este partido una ventaja decisiva sobre el Partido Liberal. Por otro lado, la débil urbanización y el surgimiento de nuevos actores sociales permitieron el surgimiento de partidos nuevos que sin embargo debieron aliarse al Partido Liberal para poder hacer frente a la formidable estructura y los inmensos recursos económicos de que disponía el Partido Colorado para mantenerse en el poder. No fue sino en 2008, casi veinte años después de iniciada la transición a la democracia que el espectro no colorado logró acceder al poder por medios electorales.

A medida que se acercaban las elecciones municipales las fuerzas del gobierno se seguían disputando posiciones de poder y acercaban a Lugo sus críticas a quienes se desempeñaban en dichos cargos y sus candidatos a reemplazarlos. Mientras tanto, el Partido Colorado aprovechó para reagruparse con miras a las elecciones municipales de noviembre de 2010. Desprovistos de acceso a las principales arcas del Estado recurrieron a un mecenas, Horacio Cartes, empresario de frontera que realizó una fortuna incalculable en las últimas décadas. El proyecto consistía en convertirlo en candidato presidencial para lo cual el debería generar los recursos que permitieran al Partido Colorado recuperarse en las elecciones municipales y posicionarse así para recuperar el poder en 2013.

El primer paso fue la elección municipal y el resultado fue presentado como un triunfo contundente del Partido Colorado que logró conquistar o reconquistar las principales intendencias del país. Efectivamente, si observamos el número de votos para intendentes por partido constatamos que el Partido Colorado obtuvo una cómoda mayoría. Pero aun así, si sumamos a los votos del PLRA los de los demás partidos se alcanza 47,58 por ciento.

Por otro lado, si observamos la tendencia vemos que el número de intendentes electos por el Partido Colorado viene descendiendo y el número del PLRA ascendiendo.

De la misma manera, si analizamos los resultados electorales para concejales municipales observamos que el Partido Colorado obtuvo 48 por ciento de los concejales y los demás partidos juntos el 52 por ciento.

Cuadro 5: Porcentaje de votos para intendentes municipales - 2010

\begin{tabular}{ccccccc}
\hline ANR & PLRA & UNACE & PPQ & FG & M20A & PEN \\
\hline 47,28 & 34,02 & 4,51 & 6,42 & 1,67 & 0,68 & 0,28 \\
\hline
\end{tabular}

Fuente: Tribunal Superior de Justicia Electoral, Elecciones Municipales. Memoria y Estadísticas, 2010. 
Cuadro 6: Número y porcentaje de intendentes electos ANR y PLRA

\begin{tabular}{lccccc}
\hline & ANR & $\%$ & PLRA & $\%$ & Total \\
\hline 2001 & 146 & $65,47 \%$ & 66 & $29,60 \%$ & 223 \\
2006 & 152 & $66,09 \%$ & 68 & $29,57 \%$ & 230 \\
2010 & 135 & $58,70 \%$ & 86 & $37,39 \%$ & 238 \\
\hline
\end{tabular}

Fuente: Tribunal Superior de Justicia Electoral, Elecciones Municipales. Memoria y Estadísticas, 2010.

Cuadro 7: Concejales electos por partidos - 2010

\begin{tabular}{ccccccccccccc}
\hline ANR & PLRA & UNACE & PPQ & PPT & FG & M20A & PEN & PPS & PDP & PDC & Otros & Total \\
\hline 1.212 & 813 & 133 & 58 & 39 & 38 & 27 & 26 & 14 & 12 & 2 & 149 & 2.523 \\
$48,0 \%$ & $32,2 \%$ & $5,3 \%$ & $2,3 \%$ & $1,1 \%$ & $1,5 \%$ & $1,1 \%$ & $1,0 \%$ & $0,6 \%$ & $0,5 \%$ & $0,08 \%$ & $6 \%$ & $99,5 \%$ \\
\hline
\end{tabular}

Fuente: Tribunal Superior de Justicia Electoral, Elecciones Municipales. Memoria y Estadísticas, 2010.

Sin duda el Partido Colorado hizo una buena elección, pero lo que influyó en la percepción que la misma era una victoria sin atenuantes fueron las victorias en Asunción, Ciudad del Este, y Encarnación, los tres principales centros urbanos. En Asunción la victoria se debió a la defección de una minoría del PLRA y, según algunos observadores, al voto cruzado del P-MAS, ya que ambos votaron a sus concejales pero al candidato a Intendente del Partido Colorado. En las demás la victoria fue incuestionable.

Sin embargo, lo más importante es fijar la atención en la cantidad de votos para candidatos a Juntas Municipales, que es el mejor indicador del voto partidario; el Partido Colorado obtuvo solo 39,34 por ciento de los votos y el voto sumado del PLRA y la izquierda alcanza 39,17 por ciento, es decir, se registra un empate. Los votos restantes, excluidos UNACE y PPQ, fueron para movimientos locales de difícil identificación política. ${ }^{8}$ Es obvio pues que las fuerzas del gobierno deberán conquistar los votos de PPQ para 2013, mientras que los votos de UNACE, si no se desplazan, asegurarían la victoria del proyecto progresista.

Como al final las elecciones presidenciales se definen por mayoria relativa, es entonces este 39 por ciento del Partido Colorado el que cuenta. Comparándolo con elecciones anteriores vemos que el partido Colorado obtuvo el 39\% de los votos en 1993, el 37 por ciento en 2003, y el 30 por ciento en 2008. La recuperación del caudal electoral del coloradismo es real entonces, pero sólo le ha servido para volver a los niveles de 2003,

$8 \quad$ Un estudio de Juan Carlos Duré Bañuelos estima que dichos movimientos pertenecen al espectro progresista. De ser así las fuerzas que apoyan al gobierno gozarían de una mayoría cómoda. En mi opinión, es probable sin embargo que en las elecciones generales dichos votos se distribuyan de una manera más o menos aleatoria, aunque con un sesgo de izquierda de magnitud difícil de estimar con precisión. Agradezco a Juan Carlos Duré Bañuelos haber compartido conmigo dichos cálculos. 
Cuadro 8: Total de votos y porcentajes para concejales municipales - 2010

\begin{tabular}{cccccccccc}
\hline ANR & $\%$ & PLRA & $\%$ & Izquierda & $\%$ & UNACE & $\%$ & PPQ & $\%$ \\
\hline 678,859 & 39,34 & 486,798 & 23,21 & 189,060 & 10,96 & 111,427 & 6,80 & 62,298 & 3,61 \\
\hline
\end{tabular}

Fuente: Tribunal Superior de Justicia Electoral, Elecciones Municipales. Memoria y Estadísticas, 2010.

y 1993. Esta recuperación es insuficiente para garantizar una victoria en 2013 salvo que las fuerzas progresistas vayan divididas.

\section{A LA BÚSQUEDA DE CANDIDATOS PARA 2013}

Ni bien terminaron las elecciones municipales, gobierno y oposición se enfrascaron en la difícil y dura tarea de articular sus ofertas electorales para 2013. Deseando un candidato no liberal para la Alianza, y careciendo de uno con suficiente carisma, sectores de Frente Guasu impulsaron la modificación de la Constitución para permitir la reelección de Lugo, la misma estrategia seguida por Nicanor Duarte Frutos y que le llevó a un estrepitoso fracaso y últimamente a la derrota en las elecciones de 2008.

El Frente Guasu procedió a recolectar firmas a fin de presionar al Congreso a aprobar una enmienda constitucional para dicho fin. El procedimiento de la enmienda, más sencillo que el de la reforma, solo requiere la aprobación del texto en ambas Cámaras del Congreso por una mayoría absoluta y luego su sujeción a un referendo aprobatorio. El procedimiento de la reforma, por otra parte, exige la conformidad de la mayoría absoluta de dos tercios de los miembros de cada Cámara y la convocatoria a una Convención Nacional Constituyente para su tratamiento. Pero la Constitución establece que no se utilizará el procedimiento de la enmienda sino el de la reforma para modificar, entre otros, aquellos procedimientos "que afecten el modo de elección, la composición, la duración de los mandatos o las atribuciones de cualesquiera de los poderes del Estado..."

Duarte Frutos alegó en su momento que la reelección nada tenía que ver con la duración de los mandatos y que por lo tanto podía establecerse por el camino de la enmienda. La reacción popular y social fue masiva y marcó el comienzo del fin de su intento de entronizarse y stroenizarse en el poder. Pero he aquí, que dirigentes que habían salido a las calles a oponerse a los planes de Duarte Frutos con la consigna de "Dictadura nunca Más!" encontraron súbitamente que Duarte Frutos había tenido razón y ellos estaban equivocados, ahora que el potencial beneficiario era otro. La campaña resultó un fiasco.

Lugo tuvo el buen tino de mantenerse públicamente al margen de esta iniciativa que como era de esperarse terminó en un fracaso estrepitoso, aunque algunos lo acusaron de alentarla desde las sombras. Dos de sus mejores ministros, el liberal Efraín Alegre, de Obras Públicas y Comunicaciones y el social-demócrata Rafael Filizzola, del Interior, que se opusieron a esta estrategia pero cuyas aspiraciones presidenciales eran obvias, fueron destituidos. El Frente Guasu no tuvo alternativas más que iniciar de nuevo la búsqueda de un candidato propio para competir con el PLRA. El primero fue reemplazado 
por un militar retirado, Cecilio Pérez Bordón, que ocupaba a la sazón el Ministerio de Defensa y el segundo por un ex comandante de Policía, Federico Acuna, y luego por el Senador Carlos Filizzola. Pérez Bordón fue sucedido por su viceministro Catalino Roig, otro militar retirado.

La realidad es que el caudal electoral del Frente Guasu no le permite competir con éxito con un candidato propio a menos que él mismo posea una popularidad muy superior a la de su base de apoyo político, como fue el caso de Lugo en 2008. Pero en la ausencia de un Lugo II el panorama electoral del Frente Guasu se complica. El Frente Guasu ha declarado que anunciará las precandidaturas el 20 de abril de 2012 y que de no lograrse un consenso recurrirá a un proceso similar al de "urna-delivery" a más tardar en julio. Hasta la fecha se han manejado las candidaturas de Sixto Pereira, dirigente campesino, senador y presidente del Partido Tekojoja; Esperanza Martínez, ministra de Salud y del mismo partido Miguel Ángel López Perito, Secretario General de la Presidencia y presidente del Movimiento 20 de abril; Carlos Filizzola, del Partido País Solidario; Lilian Soto, del Movimiento Feminista Kuña Pyrenda, y el General (R) Luis Bareiro Spaini, ex ministro de Defensa. Con excepción de Carlos Filizzola, ninguno de ellos supera el $1 \mathrm{o}$ 2 por ciento de apoyo en las encuestas conocidas.

Hacia finales de 2011 y de nuevo ahora se ha mencionado la posible candidatura de Mario Ferreiro, un conocido y popular hombre de televisión y radio. En 2011 él había declinado la posibilidad de ser candidato pero ahora ha afirmado que lo está pensando y se da por sentado que finalmente se presentará. Aunque las últimas encuestas no lo posicionan muy bien, es indiscutible que su persona concita simpatía, aprecio, y un grado de aceptación que podría permitir construir una candidatura competitiva.

El PLRA, por su parte, diseñó un original sistema denominado "urna delivery", procedimiento por el cual todos los potenciales candidatos competirín en una maxiencuesta y todos se comprometían a apoyar al ganador de la misma en las elecciones internas en las que se formalizaría la elección de candidatos. El universo de encuestados será de 60.000 personas extraídas al azar del padrón de electores, 60 por ciento afiliados al PLRA, 10 por ciento al Partido Colorado, 15 por ciento a los partidos del Frente Guasu, y 15 por ciento independientes. De esta manera asegurarían que su candidato tendría la capacidad de conquistar el voto no liberal. La encuesta se desarrollará con urnas en las que los seleccionados depositarán secretamente sus votos el 1 de abril. Los tres principales candidatos son Efraín Alegre, Blas Llano y Federico Franco. Las encuestas hasta la fecha predicen que el resultado seguiría el orden arriba enunciado. ${ }^{9}$ Luego se negociará con el Frente Guasu cómo dirimir el candidato de la Alianza, cualquiera que este fuese. Aún queda por definir si Rafael Filizzola, del PDP, participará en el proceso de selección y entrará a tallar una vez iniciado el proceso de negociación entre el Frente Guasu y el PLRA.

Este capítulo está aún por escribirse, y no existe guión. Es probable que los liberales, basados en su caudal electoral y en el hecho de haber aceptado la candidatura de Lugo en 
2008, reclamen que el Frente Guasu haga lo propio con el candidato liberal, reservándose la candidatura a vicepresidente. A juzgar por las declaraciones de sus voceros, el Frente no piensa ceder la candidatura presidencial. La solución sería una compulsa electoral.

De darse, ¿qué forma tomaría esta? El PLRA insistiría en una elección a padrón compartido o abierto a independientes. El aparato le da una gran ventaja. El Frente Guasu, por su parte, plantearía una encuesta, o una maxiencuesta al estilo del "urna-delivery" del 1 de abril, escenario en el que el aparato sólo sirve para controlar pero no para movilizar. Nada está claro aún. Quizás lo más importante, tampoco ha habido un debate sobre programas o políticas públicas. La plataforma electoral, como ha sido una costumbre hasta ahora en Paraguay, se construye sobre las personas.

En el Partido Colorado las cosas parecieron comenzar mejor pero están terminando peor. Los resultados electorales de las municipales fueron utilizados para crear un efecto de "marea" colorada y también para posicionar la candidatura de Cartes como inevitable. Sus opositores se alinearon en torno al grupo del ex vicepresidente Luis Castiglioni al que se sumó, haciendo de tripas corazón, su ex adversario Nicanor Duarte Frutos.

Cartes, que se había afiliado al Partido Colorado hacía menos de dos años no podía legalmente ser candidato. Tradicionales dirigentes del partido criticaron lo que llamaron la entrega del partido a un mecenas. Pero su grupo convocó a una convención, modificó el estatuto, y le abrió las puertas. Su avance parecía incontenible. En las internas para la presidencia colorada de marzo de 2011 triunfó su candidata, Lilian Samaniego, con el 44,3 por ciento de los votos. Segundo salió Castiglioni con 35,7 por ciento y tercero su ex compañero de fórmula de 2008 Javier Zacarías Irún, que tentado por Cartes había lanzado su propia candidatura y obtuvo el 14 por ciento de los votos. ${ }^{10}$

Los resultados de las internas coloradas produjeron realineamientos importantes. Castiglioni declinó su ambición presidencial a favor de Zacarías Irún y Duarte Frutos abandonó dicho grupo y pasó a apoyar a Cartes. El avance de Cartes continuó y más dirigentes se fueron sumando a su grupo hasta que a fines de 2011 su movimiento, denominado Honor Colorado (HC también las iniciales del candidato), empezó a resquebrajarse. El síndrome de las grandes coaliciones empezó a corroer sus bases, puesto que si bien todos lo apoyaban como candidato presidencial sus seguidores se disputaban entre sí una no muy sorda lucha por las candidaturas de Vicepresidente, Senadores, Diputados y Gobernadores.

A la fecha ya se ha producido la salida/expulsión del senador Juan Carlos Galaverna, uno de sus primeros y más importantes seguidores. Al mismo tiempo Lilian Samaniego, que había acordado el apoyo de Cartes a su candidatura partidaria a cambio del respaldo de esta a la candidatura del presidenciable, se estaría presentando como candidata presidencial, con Eleazar Salemma, un empresario de supermercados que presentó y luego retiró su candidatura presidencial. En contrapartida, Cartes logró el apoyo del candidato Arnoldo Wiens, un ex pastor menonita que había logrado posicionarse con 
un sólido 10 por ciento de simpatía en las encuestas y que atraía principalmente el voto de los colorados no muy cercanos al aparato partidario.

Según las últimas encuestas Cartes y Zacarías Irún se encuentran en una suerte de empate técnico con un respaldo de poco más de un tercio del electorado colorado cada uno, mientras Samaniego capta la simpatía de un 15 por ciento del electorado. ${ }^{11}$ Se ha pasado así de un escenario con Cartes como el inevitable ganador a un escenario muy competitivo en el que el triunfador dependerá de las jugadas a realizarse el resto del año 2012.

Finalmente, queda por verse qué postura tomarán UNACE y PPQ. El primero ha planteado siempre su interés en una alianza con el PLRA, pero el Frente Guasu se ha opuesto. Es posible que finalmente se juegue de nuevo por la candidatura de Lino Oviedo, en especial teniendo en cuenta que recientemente ha sufrido la defección de algunos de sus diputados más mediáticos. En este contexto a UNACE le convendría fortalecer su propia base de apoyo y negociar luego con quien gane la presidencia. Esta estrategia, sin duda la más conveniente para UNACE, es también la que más conviene a la Alianza puesto que los votos de UNACE en ausencia de su líder migrarían al Partido Colorado en mayor medida que a la Alianza.

El candidato de PPQ, Miguel Carrizosa, ha venido manteniendo una posición ambigua. Después de haber sido derrotado dos veces como candidato a Intendente de Asunción, Carrizosa ha capitalizado la imagen de los congresistas de PPQ que han mantenido una postura más coherente que la mayoría de sus pares. Esto es reconocido por un sector del electorado, pero minoritario, y no basta para embarcarse en una campaña presidencial que no sea puramente testimonial.

En los últimos tiempos ha habido un extraño acercamiento al Partido Colorado en defensa de los "derechos de propiedad" supuestamente amenazados por los carperos en Nacunday y una crítica constante al gobierno de Lugo por su supuesta debilidad en el combate al EPP que ha venido realizando una serie de acciones violentas de poca monta y mucho impacto. Pero los dirigentes del PPQ saben muy bien que una alianza electoral con el Partido Colorado es firmar ellos mismos su acta de defunción. Queda por ver entonces, si en el contexto de la polarización que con certeza producirá la contienda de 2013, PPQ volverá a jugar el partido en solitario, como en 2008, o tratará de negociar un acuerdo programático con la Alianza.

\section{EL BACKGROUND INSTITUCIONAL}

En contraste con este movido escenario político el background institucional ha permanecido, en lo esencial, estable. Los poderes del Estado continúan consolidando cada uno tanto poder como les resulta posible en su ámbito, sin interferir -por no querer o por no poder- en las esferas jurisdiccionales de los demás, excepto en el 
caso de los órganos multipoderes como el Consejo de la Magistratura y el Jurado de Enjuiciamiento de Magistrados. Aunque esto no es en sí cuestionable, en alguno casos llevan al quiebre de la unidad administrativa del Estado. Por ejemplo, el Poder Judicial y el Poder Legislativo se han declarado "autárquicos" presupuestariamente y proyectan y administran sus presupuestos al margen del órgano constitucional correspondiente, el Ministerio de Hacienda.

Las representaciones parlamentarias permanecen estables, con movimientos al interior de las representaciones partidarias pero sin repercutir sobre el balance de mayorías y minorías de oposición y gobierno. La excepción es la defección de tres diputados de UNACE que pasaron a integrar la bancada colorada y un senador del PDP que se integró al PPS.

El cuadro que sigue muestra la distribución de fuerzas.

Distribución de bancas en el Congreso

\begin{tabular}{lcc}
\hline Partidos & Senadores & Diputados \\
\hline ARN & 15 & $33(+3)$ \\
Bancada A & 9 & 9 \\
Bancada B & 5 & 6 \\
Bancada C & - & 13 \\
Independientes & 2 & 5 \\
PLRA & 14 & 29 \\
BancadaA & - & 13 \\
Bancada B & - & 5 \\
Bancada C & - & 9 \\
Independientes & - & 2 \\
UNACE & 9 & $12(-3)$ \\
PPQ & 4 & 4 \\
PPS & $2(+1)$ & - \\
PPT & 1 & 1 \\
PDP & $-(-1)$ & 1 \\
\hline
\end{tabular}

Finalmente, en el terreno de la calidad de la democracia la evidencia es compleja. Por una parte, si se mide en términos de calidad de la performance de la democracia en Paraguay la conclusión es que el país continúa estancado entre los de más pobres resultados. Por ejemplo, tomando los indicadores del Governance Matters del Banco Mundial con excepción de la variable "voice and accountability" (porque mide más la democracia que su performance), Paraguay figura penúltimo, solo mejor que Venezuela.

Sin embargo, si se analiza desde el punto de vista de la satisfacción de la ciudadanía con la calidad de la democracia el resultado es más alentador. Según los datos del Américas 
Calidad de la gobernanza en América Latina - 2010

\begin{tabular}{lc}
\hline País & Promedio \\
\hline Venezuela & $-1,36$ \\
Paraguay & $-0,77$ \\
Guatemala & $-0,65$ \\
Honduras & $-0,63$ \\
Bolivia & $-0,56$ \\
Nicaragua & $-0,54$ \\
Argentina & $-0,46$ \\
Ecuador & $-0,39$ \\
Colombia & $-0,36$ \\
Perú & $-0,29$ \\
El Salvador & $-0,26$ \\
México & $-0,26$ \\
Panamá & 0,01 \\
Brasil & 0,07 \\
Costa Rica & 0,53 \\
Uruguay & 0,79 \\
Chile & 1,20 \\
\hline
\end{tabular}

Fuente: Governance Matters, 2011.

Barometer para el 2010, 49 por ciento de la población estaba satisfecha con la democracia y 63 por ciento la apoyaba, resultados razonablemente buenos. El Latinobarómetro de 2011 muestra un mejoramiento en la actitud hacia la democracia, 54 por ciento la consideran preferible, pero dicho resultado está por debajo del promedio latinoamericano que es de 58 por ciento. $Y$ aun un 25 por ciento de los paraguayos cree que un gobierno autoritario es la mejor forma de gobierno, porcentaje más alto que el promedio latinoamericano del 17 por ciento y solo superado por Honduras.

Es probable que estos resultados reflejen el hecho de que, según encuestas no públicas, el presidente Lugo cuenta con un porcentaje de aprobación de gestión cercano que oscila entre el 42 y el 48 por ciento. Estos números reflejarían pues no sólo una reacción favorable de la ciudadanía hacia su imagen de bonhomía, a diferencia de la imagen agresiva y violenta que proyectaba Nicanor Duarte Frutos, por ejemplo, sino también un reconocimiento de los avances y éxitos, reales y palpables, de un gobierno que a pesar de sus deficiencias y limitaciones -varias señaladas aquí- ha sabido convocar a muchos de los mejores hombres y mujeres de Paraguay quienes han demostrado que sí puede cambiar para mejor. 


\section{REFERENCIAS}

ABC Color, Varios números identificados en las notas al final del manuscrito.

Banco Central del Paraguay (2011). Informe Económico Preliminar 2011. Asunción, 2011.

Banco Central del Paraguay (2012). Informe de Inflación-enero 2012.

Dirección General de Estadísticas, Encuestas y Censos (DGEEC), 2010. Resultados Principales de Pobreza y Distribución del Ingreso, Asunción, Paraguay.

Dirección General de Encuestas, Estadísticas y Censos (DGEEC), Principales Indicadores ECE (Encuesta Continua de Empleo). Tercer trimestre 2011, Asunción, Paraguay, 2011.

Kaufmann, Aart Kraay and Massimo Mastruzzi, Governance Matters, Washington: 2010.

Ministerio de Hacienda, Informe de Gestión 2010-2011, Asunción, Paraguay, MH, 2012.

Mitchell Selligson and Smith, A.E., eds, The Political Culture of Democracy, 2010. Democratic Consolidation in the Americas in Hard Times, Nashville: Vanderbilt University, 2011.

Tribunal Superior de Justicia Electoral, Elecciones Municipales. Memoria y Estadísticas, 2010, Asunción, Paraguay, TSJE, 2011.

Última Hora, Varios números identificados en las notas al final del manuscrito. 